\title{
High D dimers and low global fibrinolysis coexist in COVID19 patients: what is going on in there?
}

\author{
C. Ibañez ${ }^{1} \cdot$ J. Perdomo $^{1} \cdot$ A. Calvo ${ }^{1} \cdot$ C. Ferrando ${ }^{1} \cdot$ J. C. Reverter ${ }^{2} \cdot$ D. Tassies $^{2} \cdot$ A. Blasi $^{3}$ (D)
}

Accepted: 8 July 2020 / Published online: 15 July 2020

(c) Springer Science+Business Media, LLC, part of Springer Nature 2020

\begin{abstract}
Backgroud COVID-19 coagulopathy linked to increased D-dimer levels has been associated with high mortality (Fei $Z$ et al. in Clinical course and risk factors for mortality of adult inpatients with COVID-19 in Wuhan, China: a retrospective cohort study. Lancet (London, England) 395(10229):1054-62, 2020). While D-dimer is accepted as a disseminated intravascular coagulation marker, rotational thromboelastometry (ROTEM) also detects fibrinolysis (Wright FL et al. in Fibrinolysis shutdown correlates to thromboembolic events in severe COVID-19 infection. J Am Coll Surg (2020). Available from https:// pubmed.ncbi.nlm.nih.gov/32422349/ [cited 14 Jun 2020]; Schmitt FCF et al. in Acute fibrinolysis shutdown occurs early in septic shock and is associated with increased morbidity and mortality: results of an observational pilot study. Ann Intensive Care 9(1):19, 2019). We describe the ROTEM profile in severely ill COVID-19 patients and compare it with the standard laboratory coagulation test.

Methods Adult patients diagnosed with COVID-19 admitted to the ICU were prospectively enrolled after Ethics Committee approval (HCB/2020/0371). All patients received venous thromboembolism prophylaxis; those on therapeutic anticoagulation were excluded. The standard laboratory coagulation test and ROTEM were performed simultaneously at $24-48 \mathrm{~h}$ after ICU admission. Sequential organ failure assessment (SOFA), disseminated intravascular coagulation (DIC) and sepsis-induced coagulopathy (SIC) scores were calculated at sample collection.

Results Nineteen patients were included with median SOFA-score of 4 (2-6), DIC-score of 1 (0-3) and SIC-score of 1.8 (0.9). Median fibrinogen, D-dimer levels and platelet count were 6.2 $(4.8-7.6 \mathrm{~g} / \mathrm{L}), 1000(600-4200 \mathrm{ng} / \mathrm{ml})$ and $236(136-$ $36410^{9} / \mathrm{L}$ ), respectively. Clot firmness was above the normal range in the EXTEM and FIBTEM tests while clot lysis was decreased. There was no significant correlation between ROTEM or D-dimer parameters and the SOFA score.

Conclusion In COVID-19 patients, the ROTEM pattern was characterized by a hypercoagulable state with decreased fibrinolytic capacity despite a paradoxical increase in D-dimer levels. We suggest that, in COVID-19 patients, the lungs could be the main source of D-dimer, while a systemic hypofibrinolytic state coexists. This hypothesis should be confirmed by future studies.
\end{abstract}

Keywords Thromboelastometry $\cdot$ Fibrinolysis · Coagulation $\cdot$ COVID

\section{Highlights}

J.C. Reverterh, D. Tassiesh, and A. Blasia have shared the senior authorship.

A. Blasi

ablasi@clinic.cat

1 Anesthesia Department, Hospital Clinic de Barcelona, Barcelona, Spain

2 Hemostasis Department, IDIBAPS, Hospital Clinic de Barcelona, Barcelona, Spain

3 Anesthesia Department, IDIBAPS, Hospital Clinic de Barcelona, Barcelona, Spain
- Description of thromboelastometry profile of patients with COVID 19.

- Discussion of the correlation between the thromboelastometry profile and standard laboratory coagulation test results, and the possible pathophysiological hypothesis behind it.

- Bottom line for management strategies in patients with COVID 19. 


\section{Background}

Coagulopathy associated with COVID 19 has been related to significant mortality. Coagulopathy observed in severe illness has been linked with high mortality, and patients who died developed disseminated intravascular coagulation (DIC) and it has been hypothesized, based on coagulation laboratory tests [1]. Although hyperfibrinolysis is associated with a doubling of mortality in septic criticallyill patients, fibrinolysis shutdown, an acute impairment of fibrinolysis, has also been recognized as a risk factor for increased mortality in these patients [2,3].

Both hyperfibrinolysis and fibrinolysis shutdown can be assessed by thromboelastometry, a viscoelastic test that has been used as point-of-care for bleeding risk and hemostatic therapy guidance in critically-ill patients. Furthermore, its potential role in predicting mortality in septic critically-ill patients has recently been reported $[4,5]$.

Our aim was to describe the COVID-19 thromboelastometry profile using ROTEM sigma and compare it with standard laboratory findings in critically-ill patients from a Spanish tertiary hospital.

\section{Methods}

Adult patients diagnosed with COVID19 admitted to the intensive care unit (ICU) of the Hospital Clinic of Barcelona between April 5 and April 16, 2020 were prospectively enrolled. The study was approved by the Ethics Committee (HCB/2020/0371). In all cases, consent was obtained by phone from the patient or one relative and in the presence of an independent witness not related to the study. A document with the information of the patient, relative and witness was made and kept for every patient. Patients on anticoagulant treatment were excluded. Thromboprophylaxis was given according to the local protocol (enoxaparin 40-60 mg/day).

Blood citrated samples were obtained specifically for the study from 19 patients in vacutainer tubes $(0.129 \mathrm{M})$ from an arterial line or central or peripheral venous catheter. The laboratory standard coagulation test and thromboelastometry were performed simultaneously within the first hour after extraction and 24-48 $\mathrm{h}$ after ICU admission.

Demographics were recorded. Organ failure was assessed using sequential organ failure assessment (SOFA) and disseminated intravascular coagulation scores (DIC and SIC score) were calculated at blood sampling $[6,7]$. Data on hemorrhagic or thrombotic events and transfusion were collected throughout hospitalization for later assessment [8].

Thromboelastometry was performed with a point-ofcare ROTEM sigma device (TEM International, Munich,
Germany). Intrinsic coagulation pathway (INTEM) and extrinsic coagulation pathway (EXTEM) and fibrinogen function (FIBTEM) were assessed. Lysis at 30 and at 60 min (Ly60, Ly30) indicated the percentage reduction from maximum clotting firmness (MCF) at 30 or $60 \mathrm{~min}$, and was used to evaluate clot lysis, as previously described [9].

\section{Statistical analysis}

The results are presented as median and interquartile range for continuous variables and percentages for categorical variables. The SIC score was expressed as mean and standard deviation (SD). The Mann Whitney and Chi square tests were used for comparison, as needed. All analyses were performed using the Statistical Package for Social Sciences version 22.0 (SPSS, Chicago, IL), and two-tailed levels of significance were established at $5 \%$.

\section{Results and discussion}

Nineteen ICU patients were included. Baseline patient characteristics are shown in Table 1. Sixty-one percent were male, the median age was 60 years, and the BMI was above the normal range in $80 \%$. The most frequent comorbidity was hypertension (47\%). The median SOFA score was 4 [2-6] mainly due to respiratory failure. Thromboprophylaxis with enoxaparin was regularly administered to all patients except one patient who had chronic liver disease and died two days after admission due to acute respiratory failure.

At admission, high levels of fibrinogen and $\mathrm{D}$ dimer were observed, with median values of $6.2(4.8-7.6) \mathrm{g} / \mathrm{L}$ and 1000 (600-4200) ng/mL, respectively. Platelet count was preserved in most patients: mean $236\left(136-36410^{9} / \mathrm{L}\right)$ (Table 1). Thromboelastometry data showed clot firmness in the EXTEM and FIBTEM tests above the normal range, pointing to activation of coagulation. Concomitantly, clot lysis was decreased compared with reported levels in healthy controls $[5,10]$. There were no differences in thromboelastometry parameters, nor in D dimer levels between patients below/above the median SOFA value. A lower platelet count was seen in patients with SOFA $>4$. Patients were followed for 60 days. Two months after study initiation, 4 patients had died and 3 remained hospitalized. The cause of death was respiratory complications in 2 patients and multiorgan failure in 2 . There were no deaths attributed to evident thrombotic complications or bleeding. Two patients had bleeding: one required transfusion of 2 units of red blood cells. Five patients had thrombotic complications ( 2 deep vein thrombosis, 2 pulmonary embolism and 1 myocardial ischemia) and two patients had both bleeding and thrombotic complications. 
Table 1 Patient characteristics, $\mathrm{n}=19$

\begin{tabular}{|c|c|}
\hline Age, years & $61(55-73)$ \\
\hline Sex, male & $10(53 \%)$ \\
\hline BMI, kg/m² (18.5-25) & $28(27-32)$ \\
\hline $\mathrm{BMI}>25$ & $15(80 \%)$ \\
\hline \multicolumn{2}{|l|}{ Chronic disease, $\mathrm{n}(\%)$} \\
\hline Hypertension & $10(47)$ \\
\hline Diabetes mellitus & $4(19)$ \\
\hline Chronic obstructive pulmonary disease & $2(10)$ \\
\hline Chronic renal disease & $2(10)$ \\
\hline Chronic vascular disease & $3(15)$ \\
\hline Cancer & 0 \\
\hline SOFA score & $4(2-6)$ \\
\hline DIC score & $1(0-3)$ \\
\hline SIC score & $1.8(0.9)$ \\
\hline Enoxaparin (daily doses), $\mathrm{mg}$ & $60(40-80)$ \\
\hline Hemoglobin, g/L (130-170) & $120(110-140)$ \\
\hline Leukocytes, $\times 10^{9} / \mathrm{L}(4.00-11.00)$ & $9240(5860-13500)$ \\
\hline Creatinine, mg/dL $(0.30-1,30)$ & $0.9(0.7-2.0)$ \\
\hline \multicolumn{2}{|l|}{ Coagulation laboratory tests } \\
\hline Prothrombin time, s (9.9-13.6) & $12.7(12.2-13.3)$ \\
\hline Activated thromboplastin time, s (23.5-32.5) & $30(26-31)$ \\
\hline Platelets, $\times 10^{9} / \mathrm{L}(130-400)$ & $236(136-364)$ \\
\hline Fibrinogen, g/L (1.5-4.5) & $6.2(4.8-7.6)$ \\
\hline D dimer, ng/mL $(<500)$ & $1000(600-4200)$ \\
\hline \multicolumn{2}{|l|}{ ROTEM (in parentheses, normal range) } \\
\hline CT EXTEM (38-79), s & $78(63-91)$ \\
\hline CFT EXTEM (34-150), s & $41(40-53)$ \\
\hline MCF EXTEM (50-72), mm & $74(71-76)$ \\
\hline CT INTEM (100-240), s & $173(163-201)$ \\
\hline CFT INTEM (30-110), s & $51(44-64)$ \\
\hline MCF INTEM (50-72), mm & $70(67-73)$ \\
\hline FIBTEM MCF (9-25), mm & $30(24-34)$ \\
\hline LY30 EXTEM (>95\%) & $100(100-100)$ \\
\hline LY60 EXTEM (> 85\%) & $99(97-100)$ \\
\hline LY30 FIBTEM (> 95\%) & $100(100-100)$ \\
\hline LY 60 FIBTEM (> 85\%) & $100(100-100)$ \\
\hline
\end{tabular}

The results are presented as median (interquartile range) for continuous variables and percentages for categorical variables. SOFA sequential organ failure assessment, DIC score disseminated intravascular coagulation, SIC score sepsis induced coagulopathy, $B M I$ body mass index. The SIC score was expressed as mean and SD. $C T$ clotting time, $C F T$ clotting formation time, $M C F$ maximum clot firmness, LY30 lysis at $30 \mathrm{~min}$, LY60 lysis at $60 \mathrm{~min}$, EXTEM extrinsic coagulation pathway, INTEM intrinsic coagulation pathway, FIBTEM fibrinogen function

All patients who died and 4 out of 5 patients who had thrombotic events showed Ly60 100\% in the EXTEM and FIBTEM tests.

To determine whether the thromboelastometry pattern was stable or changes in a short period of time we repeated it in 5 randomly-chosen patients one week after the first sampling. No significant differences were observed between the two time points (Mann-Whitney U test for paired samples).

Patients with COVID19 showed a hypercoagulable state with a thromboelastometry pattern mainly characterized by decreased fibrinolytic capacity together with a paradoxical increase in D dimer levels, typically seen in hyperfibrinolysis. The decreased fibrinolytic capacity (fibrinolysis shutdown), defined as LY30 EXTEM 100 (100-100)\% and LY60 EXTEM 99 (97-100) observed has consistently been reported in septic and non-septic patients with early DIC. Fuat et al. reported that the fibrinolytic system function is markedly inhibited in severe sepsis and is associated with organ disfunction [11, 12].

Conversely, Panigada et al. [13], performing thromboelastography (TEG) in intubated patients with COVID19 found normal fibrinolytic capacity with values very close to the limit of hyperfibrinolysis. Additionally, although several criteria have been used to define the hypercoagulable profile in ROTEM/TEG devices [14, 15], Panigada et al described hypercoagulability in parameters of clot firmness and clot formation in TEG, whereas only one criteria of hypercoagulability (increased clot firmness in both the EXTEM and FIBTEM tests) was seen in our patients on ROTEM. These discrepancies could suggest that ROTEM and TEG perform differently in assessing fibrinolytic capacity in patients with sepsis. Disease severity (not reported by Panigada et al) and the time from disease onset could account for these differences. More recently, Wright et al. [16] also identified fibrinolysis shutdown using TEG in patients with severe COVID-19, and the presence of the fibrinolysis shutdown in addition to high $\mathrm{D}$ dimer levels correlated with thromboembolic events.

However, although high levels of D dimers were found in our patients, they did not meet the criteria for DIC as traditionally defined. Prothrombin time, fibrinogen and platelet levels were not reduced, and this was reflected by low DIC and SIC scores at ICU admission: $1(0-3)$ and $1.8(0.9)$, respectively (Table 1).

Fibrinolysis shutdown has been defined as fibrinolysis cessation subsequent to initial fibrinolysis activation. This condition has been assessed by viscoelastic global tests [17] and has been widely evaluated in trauma patients. However, the explanation remains unclear. Some investigators have suggested that a high D-dimer with low fibrinolysis reflects ongoing fibrinolysis not measured by viscoelastic tests and have called this fibrinolytic shutdown "occult hyperfibrinolysis" [18]. Others have stated that, since D-dimers have a half-life of more than $12 \mathrm{~h}$, they are biomarkers of fibrinolysis activation at some point, but do not reflect current fibrinolytic activity [19]. None of these hypotheses completely explains our results. Firstly, despite the limited sensitivity for fibrinolytic activity quantification, 
thromboelastometry represents a convenient method for clot lysis assessment [20, 21]. Secondly, the prolonged D dimer half-life cannot not explain the growing and sustained levels of $\mathrm{D}$ dimer seen in Covid-19 patients, and most probably suggests maintained fibrinolysis.

We propose a different hypothesis to explain high D dimer levels in COVID-19 patients with the global viscoelastic test showing a hypofibrinolytic pattern. The hallmark of the acute lung injury seen in severe COVID19 infection is intra-alveolar fibrin deposition. The proposed mechanism of lung damage is dramatic alveolar-endothelial disruption leading to progressive endothelial pulmonary syndrome with microvascular thrombosis [13]. It is known that urokinase is produced by lung epithelial alveolar cells and, in a SARSCov infected mice model, urokinase increased dose-dependently to the amount of recombinant mouse-adapted SARSCov injected [22]. The parameters of hypofibrinolysis found by thromboelastometry in COVID-19 patients, together with the high D-dimer levels observed may suggest the idea that the lungs could potentially be the main source of D dimers and coexist with a systemic hypofibrinolytic state. Lunggenerated $\mathrm{D}$ dimers could be due to the fibrinolysis acting on intra-alveolar fibrin membranes or local microthrombi. The fibrinolysis activator in the infected lungs could be the urokinase released by damaged epithelial alveolar cells and endothelium. Therefore, both locally activated coagulation (forming polymerized fibrin) and fibrinolysis (removing the fibrin formed) would partially explain the coexistence of severe hypoxemia together with a relatively well-preserved function of the other organs. In the lungs of patients with severe COVID19, a local imbalance between increased fibrin formation and insufficiently increased fibrinolytic activity in relation to the high burden of fibrin formed could mean that fibrin cannot be properly removed, leading to clinical respiratory manifestations. This concept may open the window to therapeutic options. Wang et al. [14] reported transient oxygenation improvement in three patients with off-label intravenous administration of tPA (Alteplase). In another series, respiratory function improved in 3 out of 4 patients after the infusion of t-PA [23]. Likewise, the effect of treatment with systemic tPA on COVID-19 associated ARDS has been estimated using a probabilistic model that predicts an improvement in pulmonary function and a reduction in mortality with the salvage use of tPA in these patients [15].

This study has several limitations. It is known that ROTEM has limited sensitivity for the quantification of fibrinolytic activity and is not suitable for the detection of other haemostatic alterations such as platelet dysfunction or the endothelial role in coagulation [20]. Additionally, the small sample size and the lack of medium-long term follow up precludes any predictive role of our findings in clinicallyrelevant hemostatic complications and outcomes, although this was not the aim of the study.
Nevertheless, our results highlight the potential pathophysiological pathways involved in hypoxemia in severe COVID19, which could allow more accurate targeted therapy.

Author contributions $\mathrm{Al}$ authors contributed to the design, data collection and writing of the manuscript.

Funding No financial founding.

\section{Compliance with ethical standards}

Conflict of interest The authors declare that they have no conflict of interest

Ethical approval Ethics Committee approval HCB/2020/0371.

\section{References}

1. Fei Z et al (2020) Clinical course and risk factors for mortality of adult inpatients with COVID-19 in Wuhan, China: a retrospective cohort study. Lancet (London, England) 395(10229):1054-62 [cited 14 Jun 2020]. Available from https:// pubmed.ncbi.nlm.nih.gov/32171076/?from_single_result=Lance $\mathrm{t}+$ Lond+Engl.+2020\%3B395\%2810229\%29\%3A

2. Wright FL et al (2020) Fibrinolysis shutdown correlates to thromboembolic events in severe COVID-19 infection. J Am Coll Surg [cited 14 Jun 2020]. Available from: https://pubmed.ncbi.nlm.nih. gov/32422349/

3. Schmitt FCF, Manolov V, Morgenstern J, Fleming T, Heitmeier S, Uhle F et al (2019) Acute fibrinolysis shutdown occurs early in septic shock and is associated with increased morbidity and mortality: results of an observational pilot study. Ann Intensive Care 9(1):19

4. Stettler GR, Moore EE, Moore HB, Nunns GR, Silliman CC, Banerjee A et al (2019) Redefining postinjury fibrinolysis phenotypes using two viscoelastic assays. J Trauma Acute Care Surg 86(4):679-685

5. Kuiper GJAJM, Kleinegris MCF, van Oerle R, Spronk HMH, Lancé MD, ten Cate $\mathrm{H}$ et al (2016) Validation of a modified thromboelastometry approach to detect changes in fibrinolytic activity. Thromb\&nbsp; 14:1

6. Vincent JL, Moreno R, Takala J, Willatts S, De Mendonça A, Bruining $\mathrm{H}$ et al (1996) The SOFA (Sepsis-related Organ Failure Assessment) score to describe organ dysfunction/failure. On behalf of the Working Group on sepsis-related problems of the European Society of Intensive Care Medicine. Intensive Care Med 22(7):707-710

7. Thachil J, Tang N, Gando S, Falanga A, Cattaneo M, Levi M et al (2020) ISTH interim guidance on recognition and management of coagulopathy in COVID-19. J Thromb Haemost. https://doi. org/10.1111/jth.14810

8. Schulman S, Kearon C (2005) Definition of major bleeding in clinical investigations of antihemostatic medicinal products in non-surgical patients. J Thromb Haemost 3(4):692-694

9. Dötsch TM, Dirkmann D, Bezinover D, Hartmann M, Treckmann JW, Paul A et al (2017) Assessment of standard laboratory tests and rotational thromboelastometry for the prediction of postoperative bleeding in liver transplantation. Br J Anaesth 119(3):402-410 
10. Schenk B, Görlinger K, Treml B, Tauber H, Fries D, Niederwanger $C$ et al (2019) A comparison of the new ROTEM $\AA$ sigma with its predecessor, the ROTEMdelta. Anaesthesia 74(3):348-356

11. Carmeliet $P$ et al (1994) Physiological consequences of loss of plasminogen activator gene function in mice. Nature [cited $29 \mathrm{Apr}$ 2020]. Available from https://pubmed.ncbi.nlm.nih.gov/8133887/

12. Adamzik M, Eggmann M, Frey UH, Görlinger K, Bröcker-Preuß M, Marggraf G et al (2010) Comparison of thromboelastometry with procalcitonin, interleukin 6 , and C-reactive protein as diagnostic tests for severe sepsis in critically ill adults. Crit Care 2010;14(5):R178. Available from http://ccforum.com/conte nt/14/5/R178

13. Panigada M, Bottino N, Tagliabue P, Grasselli G, Novembrino C, Chantarangkul V et al (2020) Hypercoagulability of COVID19 patients in Intensive Care Unit: a report of thromboelastography findings and other parameters of hemostasis. J Thromb Haemost 18(7):1738-1742

14. Blasi A (2018) Coagulation profile in patients with acute on chronic liver failure (ACLF)

15. De Pietri L, Montalti R, Begliomini B, Scaglioni G, Marconi G, Reggiani A et al (2010) Thromboelastographic changes in liver and pancreatic cancer surgery: hypercoagulability, hypocoagulability or normocoagulability? Eur J Anaesthesiol 27(7):608-616

16. Wright FL, Vogler TO, Moore EE, Moore HB, Wohlauer MV, Urban S, Nydam TL, Moore PK, McIntyre RC (2020) Fibrinolysis shutdown correlates to thromboembolic events in severe COVID19 infection. Ann Oncol. https://doi.org/10.1016/j.jamcollsur g.2020.05.007

17. Moore HB et al (2019) Fibrinolysis shutdown in trauma: historical review and clinical implications. Anesth Analg [cited $29 \mathrm{Apr}$ 2020]. Available from https://pubmed.ncbi.nlm.nih.gov/31425 218/

18. Gall LS et al (2019) The S100A10 pathway mediates an occult hyperfibrinolytic subtype in trauma patients. Ann Surg [cited 29 Apr 2020]. Available from https://pubmed.ncbi.nlm.nih. gov/31082919/?from_single_result=Gall+LS\%2C+Vulli amy $+\mathrm{P} \% 2 \mathrm{C}+$ Gille spie $+\mathrm{S}+\mathrm{et}+\mathrm{al} .+\mathrm{The}+\mathrm{S} 100 \mathrm{~A} 10+$ pathw ay + mediates + an +occult + hyperfibrinolytic + subty pe+in+trauma+patients.+Ann.+Surg.+2019\&expan ded_search_query $=$ Gall $+\mathrm{LS} \% 2 \mathrm{C}+$ Vulliamy $+\mathrm{P} \% 2 \mathrm{C}+\mathrm{Gille}$ spie $+\mathrm{S}+\mathrm{et}+\mathrm{al} .+\mathrm{The}+\mathrm{S} 100 \mathrm{~A} 10+\mathrm{pathw}$ ay + media tes+an+occult+hyperfibrinolytic+subtype+in+trauma+patie nts.+Ann.+Surg.+2019

19. Rühl H etal (2015) Label-free kinetic studies of hemostasis-related biomarkers including D-dimer using autologous serum transfusion. PLoS ONE [cited 29 Apr 2020]. Available from https://pubmed.ncbi.nlm.nih.gov/26658824/?from single_result $=\mathrm{Ruhl}+\mathrm{H} \% 2 \mathrm{C}+\mathrm{Beren} \mathrm{s}+\mathrm{C} \% 2 \mathrm{C}+$ Winterhage $\mathrm{n}+\mathrm{A} \% 2 \mathrm{C}+$ Muller+J\%2C+Oldenburg $+\mathrm{J} \% 2 \mathrm{C}+$ Potzsch+B\&expan ded_search_query $=\mathrm{Ruhl}+\mathrm{H} \% 2 \mathrm{C}+\mathrm{Berens}+\mathrm{C} \% 2 \mathrm{C}+$ Winterhage $\mathrm{n}+\mathrm{A} \% 2 \mathrm{C}+$ Muller+J $\% 2 \mathrm{C}+$ Oldenburg $+\mathrm{J} \% 2 \mathrm{C}+$ Potzsch $+\mathrm{B}$

20. Scarlatescu E, Juffermans NP, Thachil J (2019) The current status of viscoelastic testing in septic coagulopathy. Thromb Res 183:146-52

21. Meizoso JP et al (2018) Increased risk of fibrinolysis shutdown among severely injured trauma patients receiving tranexamic acid. J Trauma Acute Care Surg 84(3):426-432 [cited 29 Apr 2020]. Available from https://pubmed.ncbi.nlm.nih.gov/29298240/

22. Hattori N, Sisson TH, Xu Y, Desai TJ, Simon RH (1999) Participation of urokinase-type plasminogen activator receptor in the clearance of fibrin from the lung. Am J Physiol Lung Cell Mol Physiol 277(3 21 - 3):573-579

23. Gralinski LE, Iii AB, Jeng S (2013) Mechanisms of severe acute respiratory syndrome coronavirus. MBio 4(4):1-12

24. Poor HD et al (2020) COVID-19 critical illness pathophysiology driven by diffuse pulmonary thrombi and pulmonary endothelial dysfunction responsive to thrombolysis. Clin Transl Med [cited 14 Jun 2020]. Available from https://pubmed.ncbi.nlm.nih.gov/32508 $062 /$

Publisher's Note Springer Nature remains neutral with regard to jurisdictional claims in published maps and institutional affiliations. 